\title{
The Relationship Between Number of Comorbidities and Age of Colorectal Cancer Diagnosis in US Male Veteran Population: A Single-Center Experience
}

\author{
Abdulfatah Issak ${ }^{\mathrm{a}, \mathrm{b}, \mathrm{c}, \mathrm{f}}$, Faysal Abdulle ${ }^{\mathrm{a}, \mathrm{b}}$, Kyle Porter ${ }^{\mathrm{d}}$, Sangeeta Agrawal ${ }^{\mathrm{a}}$, b, e
}

\begin{abstract}
Background: Comorbidities of tobacco and alcohol abuse and obesity are major risk factors for colon carcinogenesis. These risk factors are considered the most prevalent modifiable risk factors linked to malignancies including colorectal cancer (CRC) in both high- and low-income countries. The aim of this study was to investigate the relationship between number of comorbidities and age of CRC diagnosis in US male veteran population.
\end{abstract}

Methods: A retrospective single-center study using chart review and the International Classification of Diseases, Ninth Revision (ICD-9) codes to identify patients with a diagnosis of CRC and comorbidities of tobacco abuse, alcohol abuse, hypertension (HTN), diabetes mellitus (DM) and chronic kidney disease (CKD). The primary aim was to study effect of these comorbidities on age of CRC diagnosis. Univariable and then multivariable logistic regression models were fit to age at diagnosis for each patient variable.

Results: A total of 362 patients were included in the study. The mean age of CRC diagnosis was 66.8. Eighty percent were Caucasians, and $20 \%$ were African Americans. African Americans were diagnosed with CRC 3.8 years younger compared to Caucasians $(\mathrm{P}=0.01)$. Controlling for other variables in the multivariable model, age at CRC diagnosis was significantly lower for African Americans and for patients with higher total counts for tobacco and alcohol abuse and obesity. HTN, DM and CKD were not associated with a lower age of CRC diagnosis.

Conclusions: Tobacco and alcohol abuse and obesity have nega-

Manuscript submitted March 22, 2020, accepted April 6, 2020

${ }^{a}$ Division of Internal Medicine, Veterans Affairs, Dayton, OH, USA ${ }^{b}$ Division of Internal Medicine, Wright State University, Dayton, OH, USA

'Division of Gastroenterology and Hepatology, Metrohealth Medical Center, Case Western Reserve University, Cleveland, OH, USA

${ }^{\mathrm{d} C e n t e r}$ for Biostatistics, Department of Biomedical Informatics, College of Medicine, The Ohio State University, Columbus, OH, USA

eDivision of Gastroenterology, Veterans Affairs, Dayton, OH, USA

${ }^{f}$ Corresponding Author: Abdulfatah Issak, Division of Gastroenterology and Hepatology, Metrohealth Medical Center, Case Western Reserve University, Cleveland, OH 44106, USA. Email: Abdul.issak@gmail.com

doi: https://doi.org/10.14740/gr1280 tive cumulative effect on age of CRC diagnosis in US male veteran population. Patients with increasing number of these comorbidities are associated with significantly lower age of CRC diagnosis. It is important to identify veterans with these comorbidities and encourage CRC screening.

Keywords: Colorectal cancer; Comorbidities; Alcohol; Tobacco; Diabetes; Age of colorectal cancer diagnosis

\section{Introduction}

Colorectal cancer (CRC) is the third most common cancer diagnosis and second leading cause of cancer-related deaths among men and women in the USA [1]. Over 150,000 new cases and 50,000 deaths annually are attributed to CRC $[1,2]$. In the USA $\mathrm{CRC}$ incidence has decreased in adults older than 50 years in the last three decades [1]. This reduction is largely attributed to increased CRC screening and detection of precancerous lesions [3]. The Veterans Affairs (VA) as a healthcare entity is a network of 153 hospital facilities and 788 community-based outpatient clinics that serve US veterans [4]. Over 5 million young and aging members of the armed forces receive care from the VA healthcare system annually [5]. In addition, about $3 \%$ of US cancer diagnoses are made in the VA annually [5]. This places the VA healthcare system at the forefront of remedying morbidity and mortality related to CRC in a sizable portion of the US population.

The American Cancer Society 2018 guidelines and the United States Preventative Services Task Force (USPSTF) current CRC guidelines recommend screening colonoscopies starting at age 45 and 50 for average risk patients respectively $[6,7]$. Current guidelines do not stratify patients based on specific risk factors such as tobacco, alcohol or obesity. Genetic predisposition accounts for a small proportion of all CRC cases, and as high as $90 \%$ of CRC cases are attributed to behavioral risk factors including physical inactivity, obesity, tobacco use and alcohol consumption [8]. Some of these risk factors, namely tobacco and alcohol consumption, are prevalent in the US veteran population, potentially placing them at a higher risk for CRC compared to the general population [9]. Few studies have evaluated the combined asso- 
Table 1. Patient Characteristics and Mean Age of Diagnosis $(N=362)$

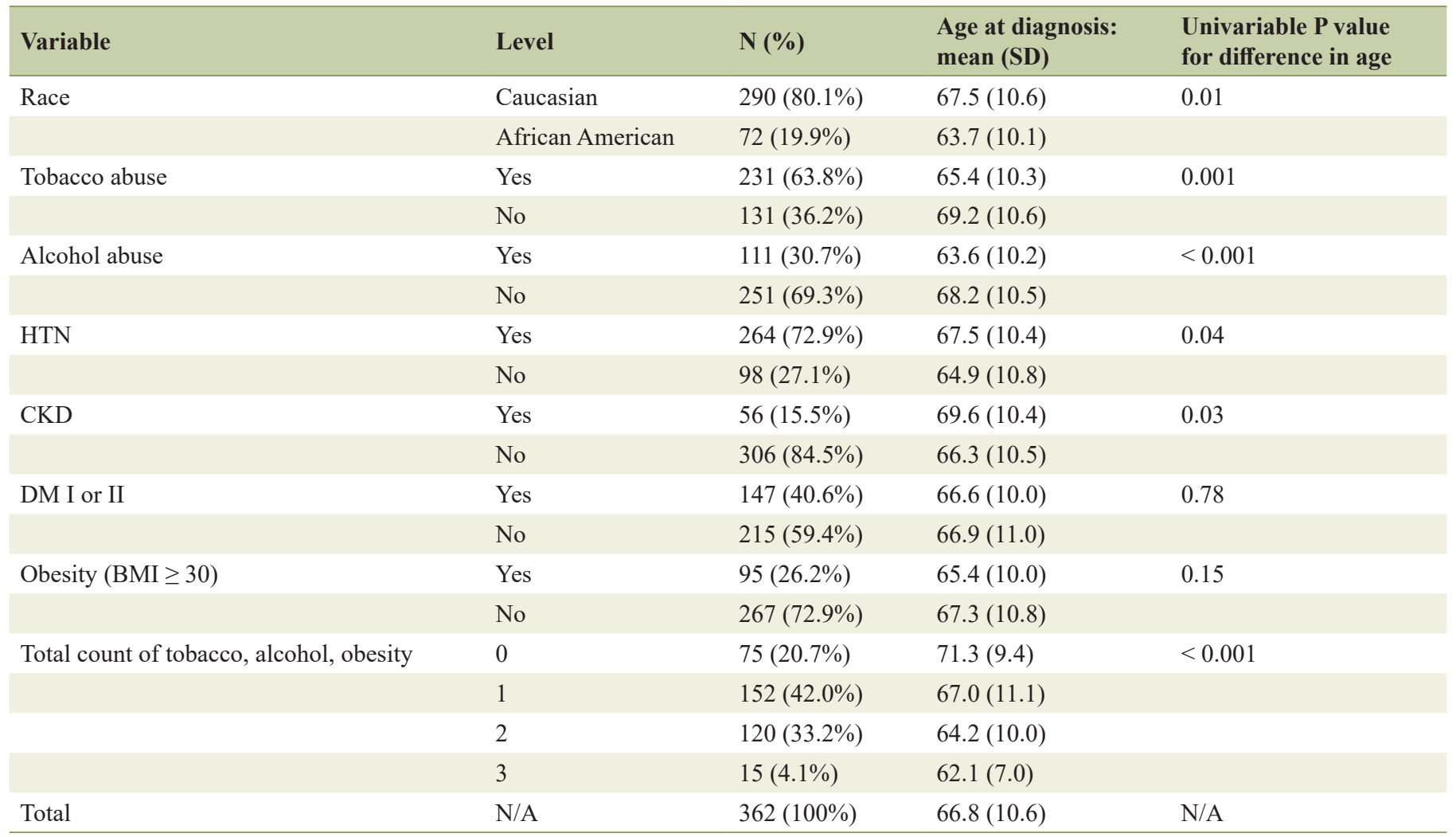

SD: standard deviation; HTN: hypertension; CKD: chronic kidney disease; DM: diabetes mellitus; N/A: not applicable.

ciation of tobacco, alcohol, and obesity and their cumulative effect as it relates to the age of CRC diagnosis in the US veteran population. The purpose of this study was to identify the prevalence of common chronic comorbidities and to assess the cumulative effect of these comorbidities on the age of CRC diagnosis.

\section{Materials and Methods}

A retrospective single-center study was performed at a large VA hospital in Dayton, Ohio. All patients with biopsy-proven CRC from January 2001 to July 2015 were included in the study. The International Classification of Diseases, Ninth Revision (ICD-9) codes $(153.9,154.1)$ were used to identify patients with a diagnosis of $\mathrm{CRC}$, and diagnoses were verified from a registry kept by the pathology department. Comorbid conditions were identified using ICD-9 codes for tobacco abuse (305.1), alcohol abuse (305.00), hypertension (HTN) (401.9), diabetes mellitus (DM) (250.00, 250.01), and chronic kidney disease (CKD) (585.9). Demographic data including name, age, gender, and race were collected. Computer-calculated body mass index (BMI) for each patient was obtained. Initial data search identified 369 patients. Seven female patients were excluded to unify patients for analysis and to make results more generalizable to the VA patient population which is predominately males. After exclusion, a total of 362 patients were included in the final analysis.

\section{Statistical analysis}

Age at diagnosis of CRC was evaluated by patient demographics and presence of comorbidities. The mean and standard deviation for the age at diagnosis is presented for each demographic and comorbidity level. Univariable linear regression models were fit to age at diagnosis for each patient variable. Three comorbidities (tobacco abuse, alcohol abuse, and obesity) were found to trend toward lower age at diagnosis. The total count of these comorbidities was identified for each patient. A multivariable linear regression model for age at diagnosis was fit including all examined variables, with tobacco abuse, alcohol abuse, and obesity included as a total count rather than individually. Interaction effects between race and comorbidities were evaluated. All statistical tests were evaluated at the $\alpha=0.05$ significance level.

The study protocol was approved by the institutional review board (IRB) at Wright State University and Dayton Veterans Affairs Medical Center.

\section{Results}

A total of 362 patients met the inclusion criteria. The incidence of each comorbidity, patient demographics and clinical charac- 
Table 2. Multivariable Linear Regression Model for Age at Diagnosis

\begin{tabular}{|c|c|c|c|}
\hline Variable & Level & Model coefficient ${ }^{a}$ (SE) & P value \\
\hline \multirow[t]{2}{*}{ Race } & African American & $-3.8(1.3)$ & 0.01 \\
\hline & White & Reference & Reference \\
\hline \multirow{2}{*}{ Total count of tobacco, alcohol, obesity } & 1 & $-4.1(1.4)$ & 0.004 \\
\hline & 2 & $-6.8(1.5)$ & $<0.001$ \\
\hline CKD & Present vs. not & $2.7(1.6)$ & 0.08 \\
\hline DM I or II & Present vs. not & $-1.5(1.1)$ & 0.20 \\
\hline
\end{tabular}

aThe adjusted mean difference in age at diagnosis (a negative coefficient indicates a lower age at diagnosis). SE: standard error of estimated coefficient; HTN: hypertension; CKD: chronic kidney disease; DM: diabetes mellitus.

teristics are listed in Table 1. The mean age of CRC diagnosis was 66.8. Eighty percent were Caucasians, and $20 \%$ were African Americans. Of the study population 63.8\% (231) had a history of tobacco use, and $30.7 \%$ (111) had a history of alcohol abuse. HTN was the most prevalent comorbidity with $72.9 \%$ (264) of patients having a diagnosis of HTN. Ninety-five $(26.2 \%)$ patients had BMI $>30$. Diabetes type I or II was present in $40.6 \%$ (147) of patients, and CKD was the least prevalent comorbidity with a prevalence of $15.5 \%$ (56). African Americans were diagnosed with CRC 3.8 years younger compared to Caucasians $(\mathrm{P}=0.01)$. Similarly, patients who use tobacco was diagnosed with CRC 3.8 years younger $(\mathrm{P}=0.001)$ compared to non-smokers. Alcohol abuse reduced the age of CRC diagnosis by 4.6 years in drinkers $(\mathrm{P}<0.001)$ compared to non-drinkers. Controlling for other variables in the multivariable model, age at CRC diagnosis was significantly lower for African Americans and for patients with higher total counts for tobacco, alcohol, and obesity (Table 2). Patients with one, two or all three diagnoses of tobacco, alcohol or obesity represented $42.0 \%$ (152), 33.2\% (120) and 4.1\% (15) respectively (Table 3). If a patient had a single diagnosis of tobacco, or alcohol or was obese, they were diagnosed with CRC 4.1 years younger $(\mathrm{P}=0.004)$. If a patient had two diagnoses of tobacco, or alcohol or was obese, they were diagnosed with CRC 6.8 years younger $(\mathrm{P}<0.001)$. If a patient had all three diagnoses of tobacco, alcohol and obesity, they were diagnosed with
CRC 8.9 years younger $(\mathrm{P}=0.002)$. Patients with diabetes had a trend toward lower CRC diagnosis; however, this was not statistically significant in multivariate analysis. Age at CRC diagnosis was significantly higher for patients with HTN alone. There were no significant interaction effects between race and any of the comorbidities.

\section{Discussion}

Analysis in our study shows a significant relationship between the number of comorbidities and age of CRC diagnosis. Obesity, history of tobacco and alcohol abuse and African American race are associated with increased CRC risk. This is consistent with known literature that shows an increased risk of $\mathrm{CRC}$ in patients of African American ancestry and those with a history of obesity, tobacco and alcohol abuse [10-19]. Our results identify a racial disparity in CRC diagnosis, and African American patients were diagnosed with $\mathrm{CRC}$ at an earlier age compared to Caucasians. This finding further supports prior studies that showed African American patients are diagnosed with $\mathrm{CRC}$ at an earlier age and have the highest mortality compared to all other racial groups $[10,11]$. While multiple studies have reported these comorbidities as risk factors for CRC, our study sought to look the combination of comorbidities and determine their association with age of CRC diagnosis. In

Table 3. Breakdown of the Tobacco, Alcohol, and Obesity Comorbidity Combinations

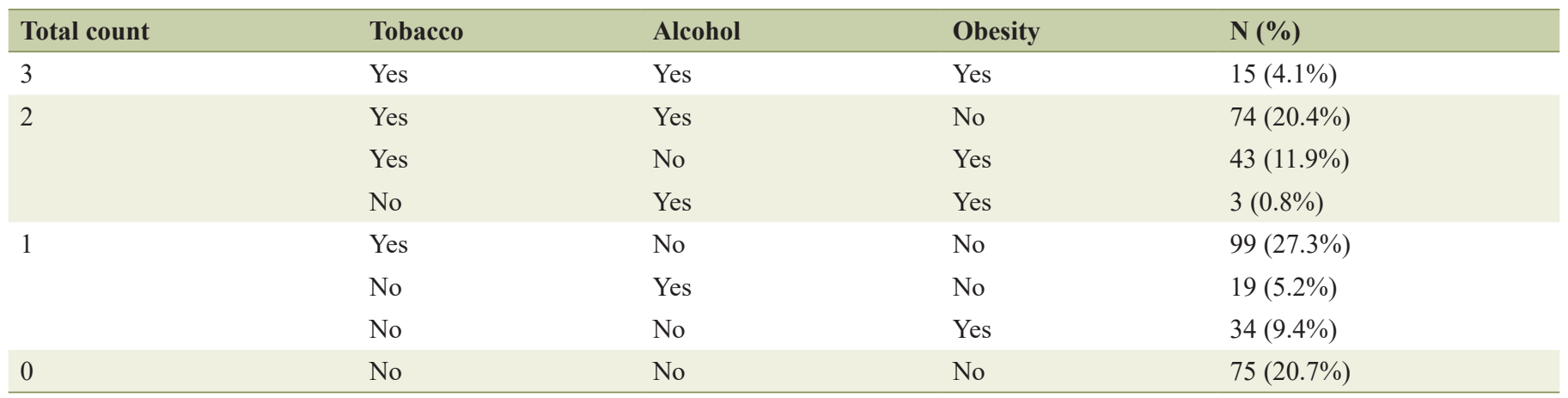




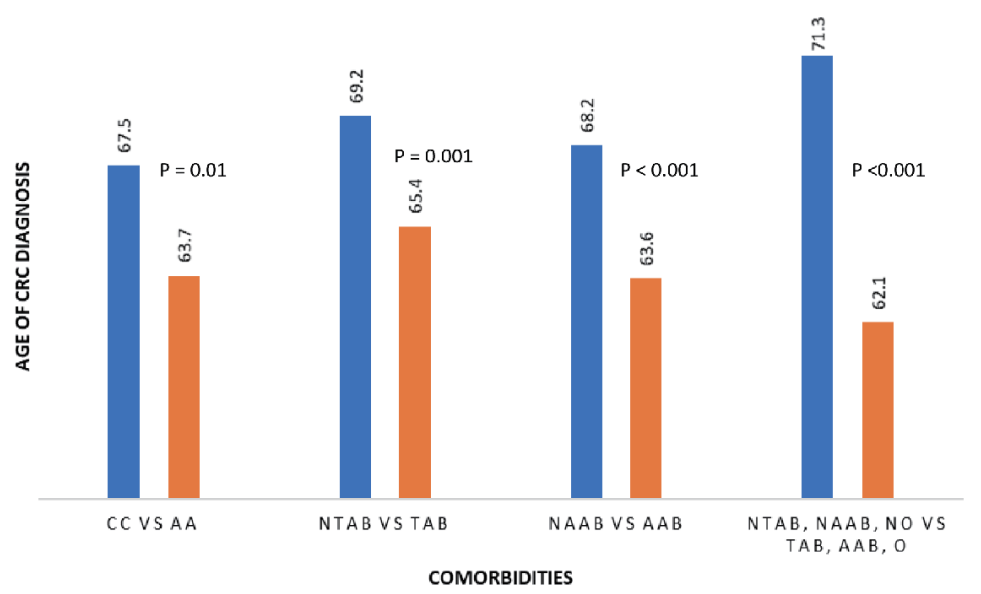

Figure 1. Relationship between race and comorbidity and age of CRC diagnosis. CC: Caucasian; AA: African American; NTAB: non-tobacco abuse; TAB: tobacco abuse; NAAB: non-alcohol abuse; AAB: alcohol abuse; NO: non-obese; O: obese; CRC: colorectal cancer.

our analyses, patients with increasing number of comorbidities are diagnosed with CRC at an earlier age. These findings are important as they provide further insight into the role of comorbidities in CRC diagnosis. Results in our study further quantify the number of years negatively affected by patients with increasing number of comorbidities. Negative effects of comorbidities on the age of CRC diagnosis remain consistent regardless of patient's race (Fig. 1).

\section{Role of alcohol and tobacco use}

Chronic alcohol consumption is well established in the literature as a major risk factor for colon carcinogenesis. It is associated with a $60 \%$ chance of $\mathrm{CRC}$ incidence $[12,13]$. In a cohort study of over 11,000 residents in a retirement community, $\mathrm{Wu}$ et al report that daily alcohol drinkers had a two-fold increase in risk of CRC [14]. In this study, researchers reported that $58 \%$ of patients diagnosed with CRC had a daily intake of alcohol containing beverage compared to $45 \%$ of non-drinkers. The risk of $\mathrm{CRC}$ is higher in those with heavy alcohol consumption. The risk is increased by $45 \%$ in those consuming at least $45 \mathrm{~g}$ (three drinks) per day [15]. However, there is no safe threshold for alcohol consumption as chronic alcohol intake even at low rates (one to three drinks daily or 10 to $40 \mathrm{~g}$ per day) is associated with 1.5 to 3.5 -fold increase in CRC risk [16]. Alcohol-related carcinogenesis stems from the metabolic byproducts of alcohol. Acetaldehyde, the main byproduct of alcohol metabolism is identified as a potent carcinogen that can promote cancer development through multiple mechanisms. Acetaldehyde is associated with interruption of DNA replication and induction of DNA damage [15]. Other alcohol metabolism enzymes, such as alcohol dehydrogenase $(\mathrm{ADH})$, aldehyde dehydrogenase (ALDH) and cytochrome P450 2E1 (CYP2E1), which are involved in the general metabolism of alcohol are also stipulated to be prone to genetic mutation in the presence of alcohol, possess harmful effects and play a role in the carcinogenesis of alcohol [15].
Current or former tobacco use is similarly established as a risk factor for CRC. CRC risk in smokers ranges from 7\% to $21 \%$ [17-19]. In one meta-analysis of 28 prospective cohort studies, the risk of CRC is increased by $21 \%$ in those who smoke at least one cigarette per day compared to never smokers [20]. In this meta-analysis, researchers compared current smokers to never smokers and noted increased CRC risk in current smokers (relative risk (RR): 1.38; 95\% confidence interval (95\% CI): 1.22 - 1.56). Another meta-analysis comparing current smokers to never smokers also reports a $17 \%(95 \% \mathrm{CI}: 0.97-1.40)$ higher risk of developing CRC and 40\% (95\% CI: $1.06-1.84)$ higher risk of CRC mortality [19]. Both meta-analyses compared former smokers to never smokers, and CRC risk appears to persist even after tobacco cessation. Comparable results of increased CRC risk were noted in both meta-analyses when comparing never smokers and former smokers (RR: 1.25; 95\% CI: 1.04 - 1.51) [19] and (RR: 1.18; 95\% CI: 1.12 - 1.25) [20] respectively. Tobacco effects on CRC risk are believed to be dose dependent and worse in those with a prolonged history of smoking or heavy smoking. In smokers of more than 30 years or more than 20 cigarettes per day, the risk of CRC is increased by $34 \%$ or $46 \%$, respectively [20]. These studies used self-reported data to establish tobacco as a significant risk factor for CRC. However, similar results were replicated in another study that used biomarkers (serum cotinine, o-cresol sulfate, and hydroxycotinine) of tobacco exposure. In a nested case-control study of 255 CRCs and 254 matched controls, Cross et al reported increased CRC risk in self-reported current smoking of any tobacco and current cigarette smoking, and further noted that individuals with detectable levels of hydroxycotinine had increased CRC risk compared to those with non-detectable levels (odds ratio (OR): 2.68; 95\% CI: 1.33 - 5.40) [20]. Moreover, smoking and alcohol use are reported as the most prevalent modifiable risk factors linked to malignancies including CRC in both high- and low-income countries [21].

Our findings are consistent with prior literature showing alcohol and tobacco use as major risk factors for CRC. Our analyses further show that as the numbers of risk factors accumulate, 
the age of CRC diagnosis is significantly reduced. This shows the overlapping contribution of these risk factors. The combination of tobacco and alcohol having the greatest negative effect on age of CRC diagnosis might be of greater importance among veterans relative to the general population, in whom substance abuse and dependence is not as prevalent. Tobacco and alcohol use are more prevalent in the veteran population [9], and it is prudent to evaluate the overall health effects of these risk factors and their role in deadly diseases such as CRC.

\section{Role of obesity and DM}

Overall obesity trend in the USA remains unfavorable; and it is estimated that $36 \%$ of the US adult population is obese [22]. An amounting body of evidence also suggests that obesity and diet rich in fats are associated with increased risk of CRC $[23,24]$. Indicators of excess body fat such as unhealthy BMI, waist-to-hip ratio and insulin resistance are linked to increased CRC risk. In a meta-analysis of 31 prospective studies, increasing BMI, waist circumference and waist-hip-ratio were associated with increased CRC risk [25]. This risk increases with worsening body habitus. Larsson et al report that a 5-unit increase in BMI is associated with a $30 \%$ increase in CRC risk in men and $12 \%$ increase in women $(\mathrm{P}<0.001)$ [25]. The mechanism by which obesity affects CRC risk remains unclear. Several pathways have been suggested including fat cells promoting proliferation of CRC cells, overexpression of fatty acid synthetase and adipokines such as adiponectin and leptin having played a role in the development of CRC [2629]. This study did not show that obese population is at high risk of CRC, probably because the number $(34,9.4 \%)$ is quite small. As well known, being overweight or obese is a high risk in both men and women of developing and dying from CRC. This conclusion needs to be demonstrated in a larger study.

In our analysis, we did not separate patients into type I or type II diabetes. No direct association between DM and colon cancer was found in the literature. Although not statistically significant in our analysis, diabetes shows a trend toward increased risk for CRC (Table 2). This is plausible given that both $\mathrm{CRC}$ and $\mathrm{DM}$ share metabolic syndrome as a common risk factor. Patients with abdominal obesity and increasing waist-to-hip-ratio are strongly associated with both DM and colon cancer [30-32].

\section{Role of HTN and CKD}

In the study subjects, HTN was noted to be the most prevalent comorbidity while CKD was the least prevalent. In our analysis, HTN and CKD were found to have a protective effect on age of CRC diagnosis. Patients with HTN and CKD were diagnosed with CRC at 3.1 and 2.7 years older respectively. HTN and CKD are closely related and are known to be risk factors for major medical conditions including coronary artery disease, myocardial infarction, heart failure and stroke with associated death and disability. However, role of HTN and $\mathrm{CKD}$ in colon carcinogenesis is not clear in the literature, and there is a paucity of literature analyzing their role in colon carcinogenesis. Given paucity of supporting literature and the nature of this single-center, retrospective study effective interpretation of this finding is limited. More studies are needed to deduce whether this is a true effect or coincidence.

Several limitations need to be considered. First, this is a retrospective chart review and we were unable to quantify amount, type, and duration of alcohol and tobacco abuse. Nonetheless, current literature points that there is no safe threshold for alcohol and tobacco consumption for cancer risk $[16,20]$. As common to any analysis that relies on self-reported data, our study is subject to potential bias and measurement errors. However, self-reported data are commonly employed in epidemiologic studies that established tobacco and alcohol risk associations with multiple cancers including lung, esophagus, oral cavity, pharynx, and larynx. Second, many of the VA patient population must undergo financial needs assessment, and those with lower levels of education might need assistance in manipulating VA benefits and coverage. Others live in rural communities with limited access to gastroenterology. These are all factors that can hinder or impact timing of age appropriate CRC screening, hence increasing their risk at baseline. Third, most VA patients are males and white, and this limits the generalizability of this study. Nonetheless, VA healthcare system is large and serves over 5 million patients annually. Therefore, results of our study are crucial as they provide insight into the disease burden in this specific patient population.

\section{Conclusions}

Our study shows that individual risk factors for $\mathrm{CRC}$ have a cumulative negative effect on age of CRC diagnosis in US veterans. Interventions to address individual CRC risk factors in US veteran population are highly needed. Cumulative negative effect of individual risk factors should be seriously considered when determining the need for CRC screening in US veteran population.

\section{Acknowledgments}

None to declare.

\section{Financial Disclosure}

Authors have no financial support to disclose for this study.

\section{Conflict of Interest}

None to declare.

\section{Informed Consent}

Given the retrospective nature of the study, informed consent 
waiver was granted by the IRB committee.

\section{Author Contributions}

Abdulfatah Issak conceived, designed and coordinated the study, wrote to the IRB, collected data, and analyzed results and wrote the manuscript. Faysal Abdulle assisted with data collection. Kyle Porter assisted with statistical analysis. Sangeeta Agrawal provided list of subjects included in the study, participated in study design and edited the manuscript.

\section{Data Availability}

The authors declare that data supporting the findings of this study are available within the article.

\section{References}

1. Siegel RL, Miller KD, Jemal A. Cancer statistics, 2016. CA Cancer J Clin. 2016;66(1):7-30.

2. Edwards BK, Ward E, Kohler BA, Eheman C, Zauber AG, Anderson RN, Jemal A, et al. Annual report to the nation on the status of cancer, 1975-2006, featuring colorectal cancer trends and impact of interventions (risk factors, screening, and treatment) to reduce future rates. Cancer. 2010;116(3):544-573.

3. Haggar FA, Boushey RP. Colorectal cancer epidemiology; incidence, mortality, survival and risk. Clinics in Colon and Rectal Surgery. 2009;22:191-196.

4. VA Benefits \& Health Care Utilization. Washington, DC, National center for Veterans Analysis and Statistics. Available at http://www.va.gov/VETDATA/PocketCard/4X6_spring10_sharepoint.pdf, April 28, 2010; accessed May 15, $201 \overline{8}$.

5. Zullig LL, Jackson GL, Dorn RA, Provenzale DT, McNeil R, Thomas CM, Kelley MJ. Cancer incidence among patients of the US Veterans Affairs Health Care System. Military medicine. 2012. 177: 6:693.

6. Wolf AMD, Fontham ETH, Church TR, Flowers CR, Guerra CE, LaMonte SJ, Etzioni R, et al. Colorectal cancer screening for average-risk adults: 2018 guidelines update from the American Cancer Society. CA Cancer J Clin. 2018;68(4):250-281.

7. www.uspreventiveservicestaskforce.org/Announcements/ News/Item/final-recommendation-statement-screeningfor-colorectal-cancer. Accessed 4/3/2018.

8. Platz EA, Willett WC, Colditz GA, Rimm EB, Spiegelman D, Giovannucci E. Proportion of colon cancer risk that might be preventable in a cohort of middle-aged US men. Cancer Causes Control. 2000;11(7):579-588.

9. Hoggatt KJ, Lehavot K, Krenek M, Schweizer CA, Simpson T. Prevalence of substance misuse among US veterans in the general population. Am J Addict. 2017;26(4):357365 .

10. Williams R, White P, Nieto J, Vieira D, Francois F, Ham- ilton F. Colorectal Cancer in African Americans: An Update. Clin Transl Gastroenterol. 2016;7(7):e185.

11. Jackson CS, Oman M, Patel AM, Vega KJ. Health disparities in colorectal cancer among racial and ethnic minorities in the United States. J Gastrointest Oncol. 2016;7(Suppl 1):S32-43.

12. Rossi M, Jahanzaib Anwar M, Usman A, Keshavarzian A, Bishehsari F. Colorectal cancer and alcohol consumption - populations to molecules. Cancers. 2018;10:38.

13. Slattery ML, Potter J, Caan B, Edwards S, Coates A, Ma $\mathrm{KN}$, Berry TD. Energy balance and colon cancer-beyond physical activity. Cancer Res. 1997;57(1):75-80.

14. Wu AH, Paganini-Hill A, Ross RK, Henderson BE. Alcohol, physical activity and other risk factors for colorectal cancer: a prospective study. Br J Cancer. 1987;55(6):687694.

15. Seitz HK, Becker P. Alcohol metabolism and cancer risk. Alcohol Res Health. 2007;30(1):38-37.

16. Scheppach W, Bingham S, Boutron-Ruault MC, Gerhardsson de Verdier M, Moreno V, Nagengast FM, Reifen R, et al. WHO consensus statement on the role of nutrition in colorectal cancer. Eur J Cancer Prev. 1999;8(1):57-62.

17. Botteri E, Iodice S, Bagnardi V, Raimondi S, Lowenfels AB, Maisonneuve P. Smoking and colorectal cancer: a meta-analysis. JAMA. 2008;300(23):2765-2778.

18. Liang PS, Chen TY, Giovannucci E. Cigarette smoking and colorectal cancer incidence and mortality: systematic review and meta-analysis. Int J Cancer. 2009;124(10):2406-2415.

19. Tsoi KK, Pau CY, Wu WK, Chan FK, Griffiths S, Sung JJ. Cigarette smoking and the risk of colorectal cancer: a meta-analysis of prospective cohort studies. Clin Gastroenterol Hepatol. 2009;7(6):682-688 e681-685.

20. Cross AJ, Boca S, Freedman ND, Caporaso NE, Huang WY, Sinha R, Sampson JN, et al. Metabolites of tobacco smoking and colorectal cancer risk. Carcinogenesis. 2014;35(7):1516-1522.

21. Danaei G, Vander Hoorn S, Lopez AD, Murray CJ, Ezzati M, Comparative Risk Assessment collaborating g. Causes of cancer in the world: comparative risk assessment of nine behavioural and environmental risk factors. Lancet. 2005;366(9499):1784-1793.

22. Ogden CL, Carroll MD, Fryar CD, Flegal KM. Prevalence of obesity among adults and youth: United States, 2011-2014. U.S. Department of Health and Human Services. Centers for Disease Control and Prevention. NCHS Data Brief. 2015;(219).

23. World Cancer Research Fund / American Institute for Cancer Research. Food, nutrition and the prevention of cancer: a global perspective. Washington, DC: American Institute for Cancer Research, 1997.

24. Terry P, Giovannucci E, Michels KB, Bergkvist L, Hansen H, Holmberg L, Wolk A. Fruit, vegetables, dietary fiber, and risk of colorectal cancer. J Natl Cancer Inst. 2001;93(7):525-533.

25. Larsson SC, Wolk A. Obesity and colon and rectal cancer risk: a meta-analysis of prospective studies. Am J Clin Nutr. 2007;86(3):556-565.

26. Amemori S, Ootani A, Aoki S, Fujise T, Shimoda R, 
Kakimoto T, Shiraishi R, et al. Adipocytes and preadipocytes promote the proliferation of colon cancer cells in vitro. Am J Physiol Gastrointest Liver Physiol. 2007;292(3):G923-929.

27. Ogino S, Kawasaki T, Ogawa A, Kirkner GJ, Loda M, Fuchs CS. Fatty acid synthase overexpression in colorectal cancer is associated with microsatellite instability, independent of $\mathrm{CpG}$ island methylator phenotype. Hum Pathol. 2007;38(6):842-849.

28. Renehan AG, Roberts DL, Dive C. Obesity and cancer: pathophysiological and biological mechanisms. Arch Physiol Biochem. 2008;114(1):71-83.

29. Stattin P, Lukanova A, Biessy C, Soderberg S, Palmqvist R, Kaaks R, Olsson T, et al. Obesity and colon cancer: does leptin provide a link? Int J Cancer. 2004;109(1):149152.

30. Kahn BB, Flier JS. Obesity and insulin resistance. J Clin Invest. 2000;106(4):473-481.

31. Pischon T, Lahmann PH, Boeing H, Friedenreich C, Norat $\mathrm{T}$, Tjonneland $\mathrm{A}$, Halkjaer $\mathrm{J}$, et al. Body size and risk of colon and rectal cancer in the European Prospective Investigation Into Cancer and Nutrition (EPIC). J Natl Cancer Inst. 2006;98(13):920-931.

32. Moore LL, Bradlee ML, Singer MR, Splansky GL, Proctor $\mathrm{MH}$, Ellison RC, Kreger BE. BMI and waist circumference as predictors of lifetime colon cancer risk in Framingham Study adults. Int J Obes Relat Metab Disord. 2004;28(4):559-567. 